\title{
El Consentimiento Informado Odontología, un Análisis Teórico.
}

\author{
Informed consent in dentistry, a theoretical analysis \\ Lilian Damián-Navarro ${ }^{1, a}$, Mirza Flores-Mori ${ }^{2, b}$, Bertha Flores-Mena ${ }^{3, c}$
}

\section{RESUMEN}

En los últimos años debido al avance de las tecnologías en el campo de la Odontoestomatología y de la salud en general, ha cobrado gran importancia la inclusión de los aspectos éticos y bioéticos que regulan la profesión, en los currículos y planes de estudio a nivel de pre y postgrado siendo EL CONSENTIMIENTO INFORMADO uno de los aspectos primordiales, ya que expresa la voluntad y autonomía que tiene el paciente para decidir sobre el tratamiento que se le va a realizar, teniendo como aspectos s éticos-legales, el Código de Ética Odontológica, Códigos Civil y Penal. En el presente artículo se hace una revisión teórica de este tema, con el objetivo de evaluar cuál es la situación en el Perú frente a su entorno en Latinoamérica.

\section{Palabras clave: CONSENTIMIENTO INFORMADO / ODONTOLOGÍA}

\section{SUMMARY}

In recent years technology advancement in Stomatology has become very important and should include ethical and bioethical aspects governing the profession, curricula and syllabi in undergraduate and postgraduate studies, being informed consent an utmost important item, as it expresses patients will and autonomy in order to decide which treatment is going to be accepted, based on ethical and legal aspects of Dental Code Ethics, Civil and Criminal Codes. In this paper a theoretical review is performed to assess the present situation in Peru, as compared with other Latin American realities.

\section{Keywords: INFORMED CONSENT / DENTISTRY}

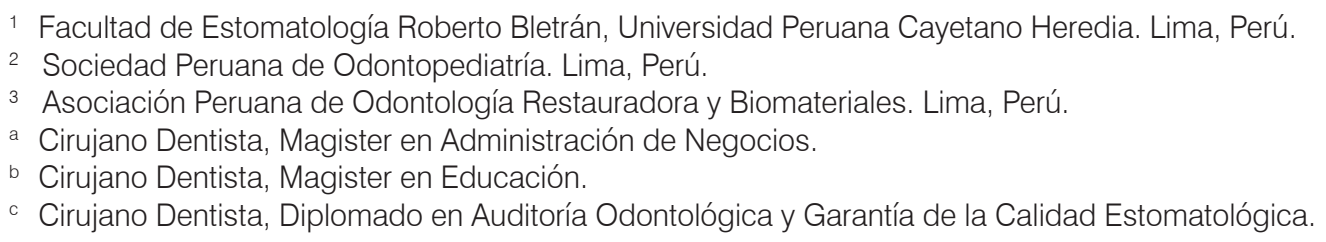




\section{INTRODUCCIÓN}

En los últimos años, los cambios producidos en el área de la salud y, en particular, en la odontología, motivaron el nacimiento de un nuevo paradigma en la relación odontólogo-paciente. El cambio del modelo paternalista al nuevo modelo autonómico imperante exige al profesional informar a su paciente todo lo concerniente a su salud bucal. Por tal motivo, surge el documento de Consentimiento Informado como instrumento escrito que pone de manifiesto las voluntades expresadas, desplegando una nueva relación de tipo contractual: el derecho del paciente a la elección responsable y el respeto a la propia libertad sobre su cuerpo y su salud $(1,2,3)$.

$\mathrm{Su}$ importancia no sólo se focaliza hacia la investigación científica, sino que alcanza también a la práctica clínica, enfatizando la importancia de la ética y la moral en los consultorios odontológicos.

En el Perú existen muy pocos estudios sobre el uso y nivel de conocimiento del consentimiento informado. Sin embargo, investigaciones realizadas sobre percepción del consentimiento informado en una clínica odontológica universitaria pública de Perú, llaman la atención acerca del desconocimiento de la existencia del consentimiento informado para la aplicación en actividades clínicas como en el proceso de investigación científica, en Odontoestomatología (4).

Otros estudios realizados en simultáneo en dos universidades que dictan la carrera de Odontología en Chile y México, señalan que existe todavía desconocimiento vago sobre conocimientos de ética y bioética del odontólogo así como del uso de la hoja del consentimiento informado para aplicación, en el desarrollo de actividades clínicas como en el proceso de investigación científica (5). Más aún, existen estudios donde se revela la carencia de información sustancial, probablemente porque no la han recibido durante su formación y, por lo tanto, tampoco saben cómo aplicarlo (6).

El Consentimiento Informado (CI) surge del cambio en la ética de la investigación biomédica, a partir de los procesos de Núremberg, que enjuiciaron la experimentación médica en humanos realizada en la Alemania nazi. Sin embargo, existen diversos antecedentes éticos del CI, desde el precepto hipocrático "primero no dañar" hasta el concepto moderno de "autonomía" de la filosofía política del siglo XVII que da origen a los principios legales ingleses, después extendidos a otros países. El concepto de autonomía del enfermo se desarrolla hasta el presente siglo $(6,7)$.

Para comprender mejor la evolución de este concepto, empezaremos por ubicar el concepto de CI en la década de los cincuenta (Miesel, et al, 1996). Posteriormente, en la Declaración de Helsinki de 1964, adoptada y modificada sucesivamente por la Asamblea Médica Mundial (1975,1985,1989,1996 y 2000), se enfatiza que la salud del paciente deberá ser la primera consideración; y que el médico actuará sólo en función del interés del paciente, tomando en cuenta que la atención médica podrá tener efectos deletéreos físicos o mentales $(6,8)$.

En Europa la Asamblea Parlamentaria del Consejo de Europa aprobó en 1976 un documento que reconocía el derecho a la información entre los derechos básicos de los enfermos. En España la Ley General de Sanidad de 1986 reconoce el derecho del enfermo al CI, el cual en uno de sus artículos establece el derecho a la libre elección entre las opciones que le presente el responsable médico de su caso para la realización de cualquier intervención (8).

El CI es una nueva forma de entender la relación médico paciente, el cual ha surgido como consecuencia de profundas transformaciones biotecnológicas de la Medicina y en la sociedad. Esta nueva forma de relación médico paciente ha llegado a constituir una exigencia ética y un derecho recientemente reconocido por las legislaciones de todos los países desarrollados $(2,9,10)$. El CI se sustenta en que al enfermo le asiste el derecho de estar informado acerca de su padecimiento, su tratamiento, terapias alternativas, riesgos y probabilidad de resultados adversos, para tomar una decisión correcta. El CI se constituye en un elemento de respeto al paciente y a su vez una nueva forma de trabajo médico el cual está regulado en el Perú por la Ley General de Salud 26842 (julio de 1997). Esta ley fue modificada por la Ley 29414 (02 de octubre de 2009); ley que establece los derechos de las personas usuarias de los servicios de salud del Perú, la cual en el artículo 15, inciso 4, contempla que la persona tiene derecho a otorgar su CI, libre y voluntario, para el procedimiento o tratamiento de salud, en especial, previa a la aplicación de cualquier procedimiento o tratamiento, cuando se trate de 
situaciones riesgosas, intervenciones quirúrgicas, anticoncepción quirúrgica o procedimientos que pueden afectar la integridad de la persona; cuando se trate de exploración, tratamiento o exhibición con fines docentes (10-13).

Sin embargo, ¿qué se entiende por consentimiento informado? La Comisión Nacional de Arbitraje Médico considera que el $\mathrm{CI}$ es el proceso por el cual el paciente competente o capaz recibe bastante la información, en términos comprensibles, que le capacita para participar en forma voluntaria, consciente y activamente en la adopción de decisiones respecto al diagnóstico y tratamiento de su enfermedad (14).

La edición de 1984 del Manual de Ética del Colegio Americano de Médicos define "el consentimiento informado consiste en la explicación a un paciente atento y normalmente competente de la naturaleza de su enfermedad, así como del balance entre los efectos de la misma y los riesgos y beneficios de los procedimientos terapéuticos recomendados, para a continuación solicitarle su aprobación para ser sometido a esos procedimientos". La presentación de la información al paciente debe ser comprensible y no sesgada; la colaboración del paciente debe ser conseguida sin coerción y el médico no debe sacar partido de su potencial dominancia psicológica del paciente (15).

La constante evolución jurídica y filosófica ha llevado a un aumento considerable de los Derechos Humanos fundamentales. En el ámbito de la ciencias de la salud, éste desarrollo ha repercutido de varias maneras impulsando el desarrollo de las cartas de los enfermos, potenciando la bioética y poniendo en crisis el modelo tradicional paternalista. De ello se deriva que el derecho al CI esté catalogado entre los más importantes derechos humanos. En este camino, se dilatan los llamados derechos de los pacientes y así se pasa de una medicina paternalista a una medicina en donde prima el principio de autonomía (16).

Actualmente no se concibe el consentimiento informado como la simple autorización que el paciente otorga al profesional de la salud para que le realice un procedimiento dado, sino como la comprensión clara, objetiva y correcta que debe tener el enfermo sobre su estado de salud, la conveniencia o inconveniencia de los procedimientos diagnósticos o terapéuticos y los beneficios y riesgos que este conlleva (16). La doctrina legal acerca del consentimiento informado identifica una norma mínima en este proceso de toma de decisiones, compartido por dentistas y pacientes. Sin embargo también vale la pena preguntarse si el CI expresa plenamente la relación ideal entre dentista y pacientes totalmente competentes (17-19).

Un requisito indispensable para la validez de CI es que prevé información clara, exhaustiva y adecuada para el paciente mismo, reconociendo que los procedimientos dentales involucran procesos con particularidades específicas como la estética, la presencia concomitantes de patologías que requieren tratamientos diversos, el proceso de una atención de larga duración y el establecimiento de una relación favorable de confianza y familiaridad con el paciente; todos ellos son aspectos que influyen en la obtención del CI (20).

Los dentistas en general, y los cirujanos maxilofaciales en particular, se han sensibilizado respecto del CI, principalmente debido al incremento en el número de pacientes que han iniciado acciones legales y a la existencia de jueces que han dictado sentencia basándose en la ausencia de tal documento $(18,21)$. Sin embargo, su verdadero valor no ha sido aún analizado ni enfatizado por los dirigentes del conjunto de profesionales en Perú. Mientras el público tiene una preocupación creciente por los derechos de los pacientes, el incremento en los costos de seguro por malpraxis genera la misma preocupación entre médicos y dentistas $(18,22)$.

Una dimensión que aún está desarrollándose es la variable edad para la aplicación del CI ya que esta responde a la legislación vigente en cada país y varía en función del rango de edad aceptada; en el caso de Chile la legislación otorga a los mayores de 14 años y menores de dieciocho la capacidad de recibir la información médica de manera directa, así como autorizar la difusión de la información a los padres o apoderados (9).

Las investigaciones a nivel internacional abordan el estudio del CI desde diversas perspectivas. En Brasil, Garbin y colaboradores (23) realizaron un estudio en una localidad cercana a Sao Paulo, para evaluar el conocimiento y práctica de los cirujanos dentistas sobre el CI, verificar la presentación y decisión del tratamiento propuestos al paciente $\mathrm{y}$ 
clase de documento utilizado por cirujanos-dentistas en la clínica odontológica. Los resultados sugieren que la mayoría de los cirujanos-dentistas presenta deficiencia al que se refiere al conocimiento acerca del CI, mientras otros desconocen totalmente el tema. Su aplicación no se realiza de manera satisfactoria, y la autorización es el documento más utilizado en la clínica odontológica. El tratamiento a realizarse depende de la decisión conjunta del paciente y profesional, presentándoselo al cliente de manera clara. De esa manera, se hacen necesarias la sensibilización y capacitación de los profesionales para la práctica del CI como rutina en la clínica odontológica.

Rogés Sánchez y colaboradores en el año 2007 (24) realizaron un estudio descriptivo, transversal, sobre los conocimientos y aplicación del consentimiento informado en 134 estomatólogos en la Atención Primaria de Salud del municipio Pinar del Río (Cuba), aplicando una encuesta y guía de observación. Se encontró que no había un conocimiento completo, ni se aplica de forma correcta el CI, siendo los elementos menos tenidos en consideración: la solicitud de autorización al paciente y la incorporación del mismo a las decisiones sobre el tratamiento a realizar.

Guirland A, en el año 2010 (25) realizó un estudio observacional descriptivo de corte transversal, aplicando un cuestionario auto-administrado, a 145 pacientes, constituido de 14 preguntas divididas en tres apartados; datos sociodemográficos, percepción y comprensión. Se observó percepción y comprensión suficiente en $62 \%$ de los participantes y el $82 \%$ de los pacientes manifestó pensar que firmar el documento eximía de responsabilidad penal al profesional. Se concluyó que no se observó significancia estadística al asociar el nivel académico con la percepción y comprensión del CI ( $\mathrm{p}=5,9, \mathrm{p}>0,005)$.

Palomer L (26) afirma que aún hay una fuerte tendencia a considerar el CI como una herramienta que protege de problemas legales y reclamos, más que como un proceso en el que se toman decisiones en forma conjunta y responsable por parte del paciente y el profesional.

Se necesita conocer, estudiar, investigar, implementary comprender el CI entre los odontólogos. Asimismo es necesario que los profesionales vean su utilidad, más allá del parámetro legal y del documento escrito, involucrándolo como parte importante de un proceso que debe ser gradual y continuo, donde se ayuda a mejorar la relación odontólogo paciente y se respeta los derechos del paciente (27).

El CI en Estomatología permite consolidar bases éticas y bioéticas en la relación odontólogo-paciente, ya que se fundamenta en el respeto a la voluntad y autonomía que tiene el paciente para decidir sobre su tratamiento.

La enseñanza acerca del uso e importancia del CI forma parte del currículo de la mayoría de Facultades de Odontología; sin embargo la forma cómo debe ser enseñada requiere un abordaje práctico y con relación a situaciones reales, las cuales el estudiante afrontará durante su vida profesional. Esto requiere la aplicación de metodologías que propicien el razonamiento crítico y la toma de decisiones, dentro de un marco sociocultural real (28).

Son diversas las instituciones de educación, como la Universidad de la República en Argentina, donde vienen evaluando la inclusión de los contenidos requeridos para adquirir competencias ético-legales en la enseñanza odontológica basada en el Proceso de CI (PCI); el objetivo del estudio fue observar los procesos de enseñanza y aprendizaje vinculados con la competencia del Proceso de Consentimiento Informado durante la formación de la carrera de grado de Odontología en la Facultad de Odontología de la Universidad de la República. En la muestra estudiada (104 estudiantes), de un total de 3120 opciones, en la dimensión de conocimientos teóricos, se contestaron correctamente $1130(36,22 \%)$. Los estudiantes no pudieron identificar conceptos básicos vinculados con cuestiones éticas y legales, teorías y reglas éticas, ni resolver teóricamente problemáticas éticoclínicas vinculadas con el PCI. Resultaron escasos los conocimientos legales referidos a la elaboración de la documentación clínica del citado proceso (29).

A nivel nacional Alfaro CLD y García RCR (26) en el año 2011 evaluaron el proceso del consentimiento informado (PCI) en pacientes atendidos en la Clínica Estomatológica de la Universidad Peruana Cayetano Heredia (UPCH), aplicaron y validaron un cuestionario que permitió evaluar el PCI en cuatro dimensiones: información, entendimiento, autonomía y valores. La muestra estuvo conformada por 90 pacientes. Los resultados reportados indican que el $85 \%$ de 
pacientes respondió que "SI" firmó el documento de CI: el entendimiento de la información y los valores del profesional fue calificado como muy favorable, mientras que la información y autonomía fue valorado sólo como favorable. La autonomía de los pacientes fue indistinta del género, edad y grado de instrucción, encontrándose diferencia significativa entre pacientes y tutores ( $\mathrm{p}=0,023)$. Se concluyó que el proceso de CI es favorable, los pacientes muestran una percepción clara y precisa del mismo, sin embargo se requiere mejorar aspectos relacionados con la información y respeto de la autonomía del paciente (30).

Son aún escasas las investigaciones en el Perú, fundamentalmente en el campo de la Odontología por lo que se hace necesario constituir un auténtico proceso de formación del odontólogo, la educación en estomatología debe sentar sus bases en valores y no básicamente un adiestramiento teórico-prácticoclínico, en donde se focaliza el aprendizaje en el número de requisitos que se debe cumplir, lo cual trae consigo dificultades en la relación del estudiante con su paciente (31).

Ante esta problemática, la profesión requiere profundizar en la teoría alrededor del CI e incursionar en la investigación cualitativa al respecto a fin de no copiar, ni "adaptar", modelos desde fuera, de poco valor en una sociedad pluriétnica y multicultural como la nuestra. Recae en las Instituciones de educación superior la responsabilidad de abordar a profundidad aspectos inherentes a los principios éticos y legales del consentimiento informado dentro de los currículos de pregrado y postgrado de odontología, para asegurar principalmente la buena relación odontólogo-paciente.

\section{CORRESPONDENCIA:}

\author{
Lilian Damián Navarro \\ Av. Honorio Delgado No 430 \\ Lima 31, Perú. \\ Correo electrónico: lilian.damian@upch.pe \\ Celular. 989083863
}

\section{REFERENCIAS BIBLIOGRÁFICAS}

1. Miguel R, Zemel M. El consentimiento informado en odontólogos residentes de la Facultad de Estomatología de la Universidad Nacional de la Plata. Acta Bioeth.
2006; 12(1):81-9.

2. Cadavid CGJ. Autonomía y consentimiento informado: principios fundamentales en bioética. Revista CES Odontología. 2005;18(2):59-64.

3. Rangel-Galvis MC. Perspectiva bioética y modelo biopsicosocial en la relación odontólogo-paciente durante la formación del estudiante en la Universidad el Bos-15 RCR. Conocimientos de ética y bioética del odontólogo en el ámbito docente. Rev Latinoam Bioetica. 2009;9(1):70-5.

5. Buendía LAL, Álvarez de la Cadena SC. Nivel de conocimiento de la bioética en carreras de odontología de dos universidades de América Latina. Acta Bioeth. 2006;12(1):41-7.

6. Garmendia HG, Vila MD, Felipe GAM, Felipe AA, Baró GMT. El consentimiento informado en el tratamiento integral del niño con fisura labio-alveolopalatina. Rev Cubana Estomatol [internet]. 2013 Mar [citado 2014 Mar 19] ; 50(1): 28-40. Disponible en: http://scielo.sld.cu/scielo.php?script=sci arttext\&pid=S0034-75072013000100003\&lng=es.

7. León FJ. Fundamentos y principios de bioética clínica, institucional y social. Acta Bioeth. 2009;15(1):70-8.

8. Zavala S, Gutiérrez W, Chiang M. Seguimiento del proceso de obtención del consentimiento informado en los participantes de protocolos de investigación. Rev Soc Peru Med Interna. 2007;20(1):10-15.

9. León CFJ. Información y consentimiento informado de menores de edad en Chile. Rev. chil. pediatr. [internet]. 2012 Abr [citado 2014 Mar 19] ; 83(2):113-116. Disponible en: http://www.scielo.cl/scielo.php?script= sci_arttext\&pid=S0370-41062012000200001\&lng=es. http://dx.doi.org/10.4067/S0370-41062012000200001.

10.Zárate CE. Los derechos de los pacientes y el Conocimiento Informado en Perú; SITUA. 2003;12(23):4-10.

11. Ley General de Salud, Ley $N^{o}$ 26842. (20 de julio de 1997).

12. Ley que establece los derechos de las personas usuarias de los servicios de salud, Ley $\mathrm{N}^{\circ}$ 29414. (30 de setiembre de 2009).

13. Rillo AG. Consentimiento informado: aspectos éticos y legislación en la odontología. Rev Hum Med [internet]. 2013 Ago [citado 2014 Mar 19; 13(2):393411. Disponible en: http://scielo.sld.cu/scielo.php? s c ri p t $=$ s c i _ a r t t e x t \& p i d = S 1727 $81202013000200007 \& \operatorname{lng}=$ es.

14. Gómez R. Consentimiento informado en odontología. Rev Asoc Dent Mex. 2007;64(5):205-7.

15. Sardiñas G, Ortega M, Morales E. Consentimiento informado. Derecho del paciente y obligación del médico contemporáneo [internet]. 2010; 2. Disponible en: http://www.monografias.com/ trabajos40/consentimiento-informado/consentimientoinformado.shtml. 
16. Cortéz CN. El pronóstico: un compromiso ético fundamental para el consentimiento informado. Revista Facultad de Odontología Universidad de Antioquia. 2006; 18(1):103-110.

17. Escobar MT. Consentimiento informado en odontología [internet]. Colombia; 2004; 1. Disponible en: http://www.encolombia.com/odontologia/foc/FocXXConsentimiento.htm.

18. Agranatti P. Bioética en odontología [internet]. Argentina; enero 2001. Disponible en: http://www. odontomarketing.com/numeros\%20anteriores/ ART_18_ENE_2001.htm

19. Izzeddin AR. El odontólogo frente al consentimiento informado: aspectos relevantes. Acta Odontológica Venezolana. [internet]. 2011 Dic [citado 2014 Mar 19]; 49(2):1-7. Disponible en: http://www.actaodontologica. com/ediciones/2011/2/pdf/art19.pdf

20. Pentón GV, Véliz AZ, Prado LB, Mary HL. La ética y la bioética. Bases del consentimiento informado en Ortodoncia: modelos de diagnóstico y evaluación. MediSur [internet]. 2009 Dic [citado 2014 Mar 19]; 7(6): 42-51. Disponible en: http://scielo. sld.cu/scielo.php?script $=$ sci_arttext $\&$ pid $=$ S 1727 897X2009000600008\&lng=es

21. Capote FJ, Castillo BE, Quiñones BA, Espino OS. El consentimiento informado en las extracciones dentarias: bases bioquímicas y su aplicación en la enfermedad cerebrovascular isquémica. MediSur [internet]. 2009 Jun [citado 2014 Mar 19]; 7(3):70-74. Disponible en: http://scielo.sld.cu/scielo.php?script=sci arttext\&pid=S1727-897X2009000300011\&lng=es.

22. Calderón MJA. El consentimiento informado y la mal praxis en odontología. Tesis de Grado de Magister en Ciencias en Bioética. Instituto Politécnico Nacional de México. 2006. [citado 2014 Mar 19]. Disponible en: h t t p : / / t e s i s.ip n. mx/d s p a c e / bi t s trea m/123456789/3255/1/ ELCONSENTIMIENTOINF.pdf

23. Garbin, CAS; Garbin, AJI; Saliba, N.A.; Zina, LG; Gonçalves, PE. El consentimiento informado en la clínica odontológica. Acta Odontológica Venezolana. 2007; 45(1):1-10.

24. Rogés SAV; Sánchez GS; Sanabria NJ.; Sosa HH; Moleiro HM. Aplicación del Consentimiento Informado por Estomatólogos en la Atención Primaria de Salud. Rev Ciencias Médicas [internet]. 2010 Mar [citado 2014 Mar 18]; 14(1): 26-36. Disponible en:http://scielo.sld.cu/scielo.php?script=sci arttext\&pid=S1561-31942010000100004\&lng=es.

25. Guirland AA. Percepción y comprensión del consentimiento informado en pacientes que acudieron a la Facultad de Odontología en el año 2010. Mem. Inst. Investig. Cienc. Salud. [internet]. 2011 [citado 20 Marzo 2014]; 9(2):43-49. Disponible en:http://scielo. iics.una.py/scielo.php?script=sci_arttext\&pid=S1812-
$95282011000200006 \& \operatorname{lng}=\mathrm{es} \& n r m=$ iso $>$ ISSN 1812 9528.

26. Palomer RL. Consentimiento informado en odontología: un análisis teórico-práctico. Acta bioeth. [internet]. 2009 [citado 2014 Mar 19];15(1): 100-105. Disponible en: http://www.scielo.cl/ scielo.php?script=sci_arttext \&pid=S 1726 569X2009000100013\&lng=es. http://dx.doi. org/10.4067/S1726-569X2009000100013.

27. Torres-Quintana MA, Romo F. Bioética y ejercicio profesional de la Odontología. Acta Bioeth. 2006; 12(1):65-74.

28. Zaror SC, Vergara GC. Ética en el currículo de las carreras de odontología. Acta bioeth. [internet]. 2008 [citado 2014 Mar 20]; 14(2): 212-218. Disponible en: $\quad$ http://www.scielo.cl/scielo.php?script=sci arttext\&pid=S1726-569X2008000200012\&lng=es. h t t p://dx.doi.org/10.4067/S 1726 569X2008000200012.

29. Miguel R; Salveraglio I.; Zemel MG: Piovesan S; Cocco LA. La competencia odontológica del proceso de consentimiento informado durante la formación de grado en Uruguay. Odontoestomatología [internet]. [citado 2014 Mar 18]. Disponible en: http://www. s c i e lo.edu.uy/s c i e lo.ph p ? s cript=s ci arttext\&pid=S1688-93392010000300005\&lng=es.

30. Alfaro CLD, García RCR. Percepción del proceso de consentimiento informado en pacientes de la Clínica Estomatológica Central de la Universidad Peruana Cayetano Heredia. Rev Estomatol Herediana. 2011;21(1):5-12.

31. Izzeddin AR, Spina M, Tosta E. Ethical dimension in dentistry practice: personalist viewpoint. Acta bioeth. [internet]. 2010 Nov [citado 2014 Mar 20]; 16(2):207210. Disponible en: http://www.scielo.cl/scielo.php? script=sci_arttext\&pid=S1726-569X2010000200014 $\& \operatorname{lng}=$ es.http://dx.doi.org/10.4067/S 1726 569X2010000200014.

Recibido : 07/10/2013

Aceptado: 13/12/2013 